\title{
Cirrus cloud occurrence as function of ambient relative humidity: a comparison of observations obtained during the INCA experiment
}

\author{
J. Ström ${ }^{1}$, M. Seifert ${ }^{1}$, B. Kärcher ${ }^{2}$, J. Ovarlez ${ }^{3}$, A. Minikin ${ }^{2}$, J.-F. Gayet ${ }^{4}$, R. Krejci ${ }^{1}$, A. Petzold ${ }^{2}$, F. Auriol ${ }^{4}$, \\ W. Haag ${ }^{2}$, R. Busen ${ }^{2}$, U. Schumann ${ }^{2}$, and H. C. Hansson ${ }^{1}$ \\ ${ }^{1}$ ITM, Air Pollution Laboratory, Stockholm University, Sweden \\ ${ }^{2}$ DLR, Institut für Physik der Atmosphäre, Oberpfaffenhofen, Germany \\ ${ }^{3}$ LMD, Ecole Polytechnique, CNRS-IPSL, Palaiseau, France \\ ${ }^{4}$ LAMP, Université Blaise Pascal, Aubiere, France
}

Received: 24 March 2003 - Published in Atmos. Chem. Phys. Discuss.: 30 June 2003

Revised: 30 September 2003 - Accepted: 15 October 2003 - Published: 27 October 2003

\begin{abstract}
Based on in-situ observations performed during the Interhemispheric differences in cirrus properties from anthropogenic emissions (INCA) experiment, we introduce and discuss the cloud presence fraction $(\mathrm{CPF})$ defined as the ratio between the number of data points determined to represent cloud at a given ambient relative humidity over ice (RHI) divided by the total number of data points at that value of RHI. The CPFs are measured with four different cloud probes. Within similar ranges of detected particle sizes and concentrations, it is shown that different cloud probes yield results that are in good agreement with each other. The CPFs taken at Southern Hemisphere (SH) and Northern Hemisphere $(\mathrm{NH})$ midlatitudes differ from each other. Above ice saturation, clouds occurred more frequently during the $\mathrm{NH}$ campaign. Local minima in the CPF as a function of RHI are interpreted as a systematic underestimation of cloud presence when cloud particles become invisible to cloud probes. Based on this interpretation, we find that clouds during the SH campaign formed preferentially at RHIs between 140 and $155 \%$, whereas clouds in the $\mathrm{NH}$ campaign formed at RHIs somewhat below $130 \%$. The data show that interstitial aerosol and ice particles coexist down to RHIs of 70-90\%, demonstrating that the ability to distinguish between different particle types in cirrus conditions depends on the sensors used to probe the aerosol/cirrus system. Observed distributions of cloud water content differ only slightly between the $\mathrm{NH}$ and SH campaigns and seem to be only weakly, if at all, affected by the freezing aerosols.
\end{abstract}

Correspondence to: J. Ström

(johan@itm.su.se)

\section{Introduction}

There is a concern that anthropogenic emissions may change the environment in a way that it could influence the frequency of occurrence and microphysical properties of cirrus clouds. An obvious anthropogenic modification of the cloud frequency of occurrence are contrails formed behind aircraft near the tropopause. Based on analyzed satellite data, Mannstein et al. (1999) and Meyer et al. (2002) deduced that linear persistent contrails cover about $0.5-0.7 \%$ of the sky at noon over Europe in the annual average. These results exemplify the possible regional effect by contrails (IPCC 1999, EC 2002).

Besides this direct contrail effect, observations indicate that indirect aerosol effects may influence the properties of high clouds as well (Ström and Ohlsson, 1998; Boucher, 1999; Kristensson et al., 2000). A pronounced indirect aerosol effect on cirrus is possible when at least two types of freezing aerosol particles compete during cloud formation (Kärcher and Lohmann, 2003): adding efficient ice nuclei to liquid aerosol particles can lead to a marked suppression of pristine ice crystal number densities, the magnitude of this effect depending on updraft speed, temperature, and number and freezing properties of the ice nuclei.

Compared to our current level of understanding of warm cloud microphysical processes, fundamental knowledge about the formation and disappearance of cirrus clouds is still lacking, as there are still open issues regarding the preferred mode of ice nucleation in cirrus clouds (DeMott, 2002) and the evaporation kinetics of small ice crystals under polluted conditions (Chen and Crutzen, 1994; Seifert et al., 2003a). The situation is complicated by the fact that the efficiency with which aerosol particles freeze is strongly linked to the dynamical conditions prevailing during ice formation (Kärcher and Ström, 2003). 
Studying a possible anthropogenic effect on clouds involves the detection of subtle but systematic differences between the properties of clouds formed in a pristine environment and those formed in a perturbed environment. The possible anthropogenic influence on warm clouds has been studied extensively and covers a range of environmental conditions in both hemispheres. However, until recently all insitu measurements of midlatitude cirrus had been performed in the Northern Hemisphere. With the project INCA (Interhemispheric differences in cirrus properties from anthropogenic emissions) the first observations of cirrus properties in the Southern Hemisphere midlatitudes became available, allowing clouds that formed under comparable meteorological conditions in two very different regions of the world to be compared with each other with an identical set of in situ instruments.

Two aircraft campaigns were performed as part of INCA, one in the Southern Hemisphere (SH) and one in the Northern Hemisphere $(\mathrm{NH})$ midlatitudes. Here the abbreviations $\mathrm{SH}$ and $\mathrm{NH}$ are used to identify the different campaigns and do not refer to hemispherically averaged properties. The first campaign based in Punta Arenas, Chile ( $\left.54^{\circ} \mathrm{S}\right)$ was performed in March and April. The second campaign based in Prestwick, Scotland $\left(55^{\circ} \mathrm{N}\right)$ was performed in September and October. Hence, the campaigns were performed in equivalent seasons in the same year 2000. For more information about the INCA experiment we refer to: http: //www.pa.op.dlr.de/inca/.

One of the first results published from INCA was a comparison of distributions of relative humidity over ice (RHI) observed during the two campaigns (Ovarlez et al., 2002). Both data sets presented a maximum in the frequency distribution around $100 \%$, but the distribution was skewed towards higher humidities in the SH data. In the present study, we investigate whether differences in the distributions of RHI are related to differences in cirrus cloud properties by comparing the occurrence of clouds as a function of RHI observed during the two campaigns. For this purpose, we define cloud occurrence as the ratio between the number of in-cloud data points versus all data points at any given RHI and call this ratio the cloud presence fraction (CPF). A substantial part of the paper deals with the question of how to decide whether a data point represents cloudy or cloud-free air.

\section{Methodology}

Although a cloud is something known to everyone, it may sometimes be difficult or even impossible to provide a simple definition for when a cloud is actually present or not. What is the minimum crystal number density or horizontal and vertical extent necessary for an ensemble of hydrometeors to be called a cloud? Is a $1 \mathrm{~m}$ thick layer or a particle number density of $1 \mathrm{~m}^{-3}$ sufficient? We can raise similar questions for observable parameters obtained by in-situ or remote sensing methods alike. Because of theses difficulties, the presence or non-presence of a cloud is usually determined by the detection limit of the particular sensor used to observe the cloud. What is interpreted as a cloud by one sensor might be interpreted as cloud-free air by another.

\subsection{Description of the cloud probes}

In this study we will make use of four different cloud sensors to investigate the presence or non-presence of cirrus clouds as function of ambient relative humidity. These instruments are the Counterflow Virtual Impactor (CVI), the PMS FSSP-300, the PMS 2D-probe, and the Polar Nephelometer. The same instruments were used in both INCA campaigns, which permits a direct comparison of the observations with respect to an unchanged payload configuration. The afore-mentioned cloud probes were mounted on the research aircraft Falcon operated by Deutsches Zentrum für Luft- und Raumfahrt (DLR). All probes have different advantages and limitations and provide information about different aspects of the cloud, as explained below. With the term "cloud particles" used hereafter we mean "particles measured in the presence of cirrus clouds".

The CVI (Ogren et al., 1985; Ström et al., 1994) has its inlet facing the direction of flight and operates by using an internal flow opposite the flight direction to prevent ambient air and small particles to enter the probe. In the upper troposphere and at typical Falcon airspeeds of $\sim 180 \mathrm{~m} \mathrm{~s}^{-1}$, this lower cut-off is approximately $5 \mu \mathrm{m}$ aerodynamic diameter. Cloud particles with these and larger sizes are mostly ice crystals, especially at the high total number concentrations $\left(>0.1 \mathrm{~cm}^{-3}\right)$ typically found in young cirrus clouds. The crystals entering the CVI are evaporated and their residues are counted using condensation particle counters. A one-toone correspondence between the number of residual particles and ice crystals is assumed, which is proven to be a valid assumption (Seifert et al., 2003b). The total particle number density captured by the CVI is an accurate measure of the total number density of ice crystals in young cirrus clouds. At $1 \mathrm{~Hz}$ data resolution, one count registered by the CVI payload corresponds to an ambient crystal number density of $\sim 0.0004 \mathrm{~cm}^{-3}$ (CVI detection limit).

The FSSP-300 probe uses the measured response from scattered light coming from particles illuminated by laser light (Baumgardner et al., 1992). To convert from the observed scattered light signals to a particle size distribution, a T-matrix calibration was used (Borrmann et al., 2000). The method yields a size classification in 32 bins between $0.37 \mu \mathrm{m}$ to $15.77 \mu \mathrm{m}$ in diameter. Cloud particles in this size range can be interstitial aerosol particles and ice crystals, especially in the sub- $\mu \mathrm{m}$ size range. Due to noise in the first 3 bins (smallest sizes), the minimum size class starts at $0.55 \mu \mathrm{m}$. At $1 \mathrm{~Hz}$ data resolution, one count registered by the FSSP-300 corresponds to a particle number density of $\sim 0.2 \mathrm{~cm}^{-3}$ (FSSP detection limit). 

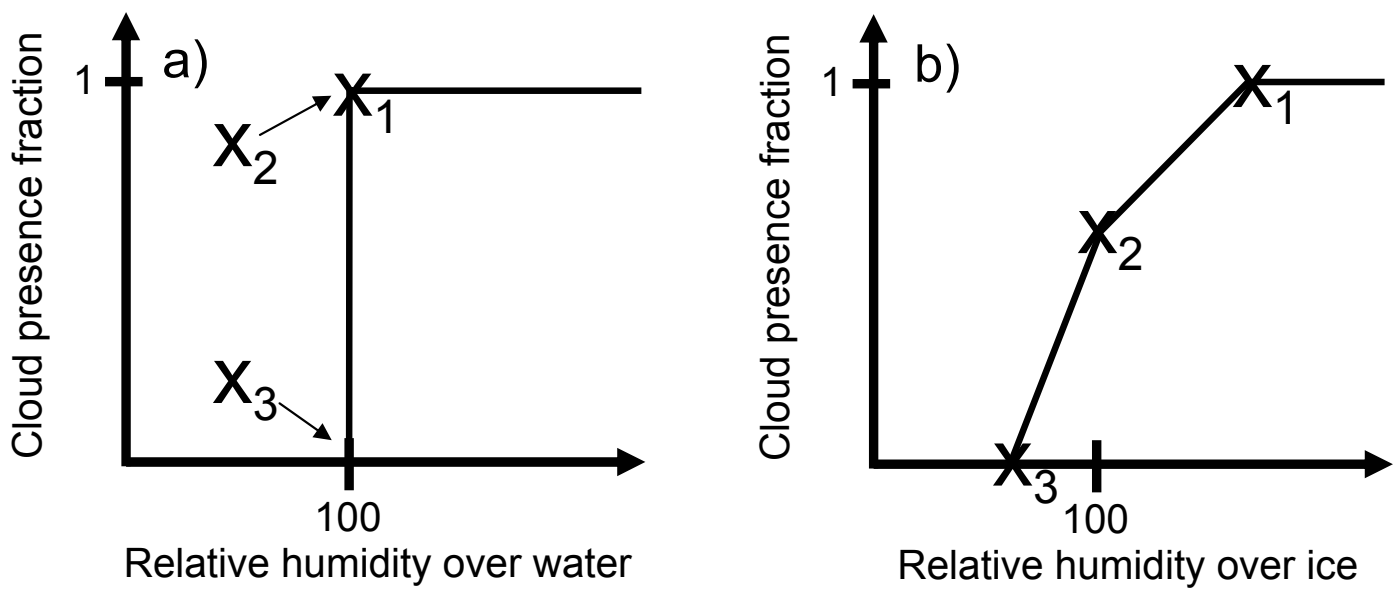

Fig. 1. Schematic illustration of the cloud presence fraction as a function of the relative humidity for liquid clouds (a) and ice clouds (b). All data points above $X_{1}$ are in-cloud data points. The point where clouds start to dissolve and where cloudy and non-cloudy air parcels coexist is marked $\mathrm{X}_{2}$ and the point where the cloud has completely disappeared is marked $\mathrm{X}_{3}$.

The PMS 2D-probe classifies the size of particles by the shadow they create when passing in front of an array of detectors illuminated by a laser. The particles are classified into 30 bins in the diameter range $25-800 \mu \mathrm{m}$. Cloud particles in this size range are exclusively water ice crystals, based on their large condensed mass. Because such large ice crystals are usually not spherical, a number of assumptions are introduced to convert from raw data to size distributions (Gayet et al., 1993). Most of the cloud water content (CWC) is located in the size range of the $2 \mathrm{D}$-probe. The ambient crystal number density corresponding to one count registered by the 2D-probe depends on the crystal size, but typically the minimum detectable number density is of the order $10^{-6} \mathrm{~cm}^{-3}$ (2D-probe detection limit).

The Polar Nephelometer measures the scattering phase function of an ensemble of cloud particles in the size range from a few $\mu \mathrm{m}$ to about $800 \mu \mathrm{m}$ diameter (Gayet et al., 1997), i.e., essentially ice crystals. A laser beam at a wavelength of $804 \mathrm{~nm}$ illuminates the ice crystals near the focal point of a paraboloidal mirror. The scattered light is detected by 44 photodiodes in angles from \pm 3.49 to $\pm 169^{\circ}$. The direct measurement of the scattering phase function provides the means to distinguish between solid and liquid (if present) phase hydrometeors and to calculate the extinction coefficient and asymmetry parameter (Auriol et al., 2001). The Polar Nephelometer provides an optical response (volume extinction) that depends on the combined effect of ice crystal size and number density.

\subsection{Relative humidity and cloud presence fraction}

Relative humidity was measured using a cryogenic frost point mirror (Ovarlez and van Velthoven, 1997). The unheated inlet, a modified Rosemount-Goodrich temperature housing, was located on the top of the fuselage. The relative humidity is determined from the Sonntag saturation vapor pressure formula (Sonntag, 1994), using the air temperature data provided by the standard instrumentation on board the Falcon. The relative uncertainty in observed relative humidity is estimated to be better than $7 \%$ ( 2 standard deviations).

The expected CPF as function of relative humidity over supercooled liquid water for warm clouds consisting of supercooled water droplets is illustrated in Fig. 1a. Such clouds form and disappear at essentially the same relative humidity marked $X_{1}$ and $X_{2}$. Actually, a small supersaturation, typically a fraction of a percent, is necessary to activate aerosol particles into cloud droplets. However, within measurement capabilities of relative humidity, the CPF can be approximated as unity at and above water saturation and zero at humidities below. Solution droplets may be in equilibrium with ambient water vapor even at relative humidities well below $100 \%$. These particles are not activated into cloud droplets in the traditional sense.

Ice clouds are more complicated than their liquid counterparts since they may form at one RHI substantially (many tens of percent) above saturation and dissolve completely at much lower (few tens of percent) values below saturation; see Sect. 3. The rate at which water molecules are transferred between the different phases at cold temperatures is slower than in warm clouds, which is why a cirrus cloud can persist for some time in air subsaturated with respect to ice and give rise to their often fuzzy appearance especially around cloud edges. A possible scenario is illustrated in Fig. $1 b$. At some relative humidity over ice, $\mathrm{X}_{1}$, ice crystals appear in any given air parcel. In this case we do not care about their size and number density, we simply acknowledge the presence of cloud. The relative humidity may still increase after crystals initially appear. This increase in relative humidity continues until the formation phase is completed and all crystals have formed. In our case this does not change 

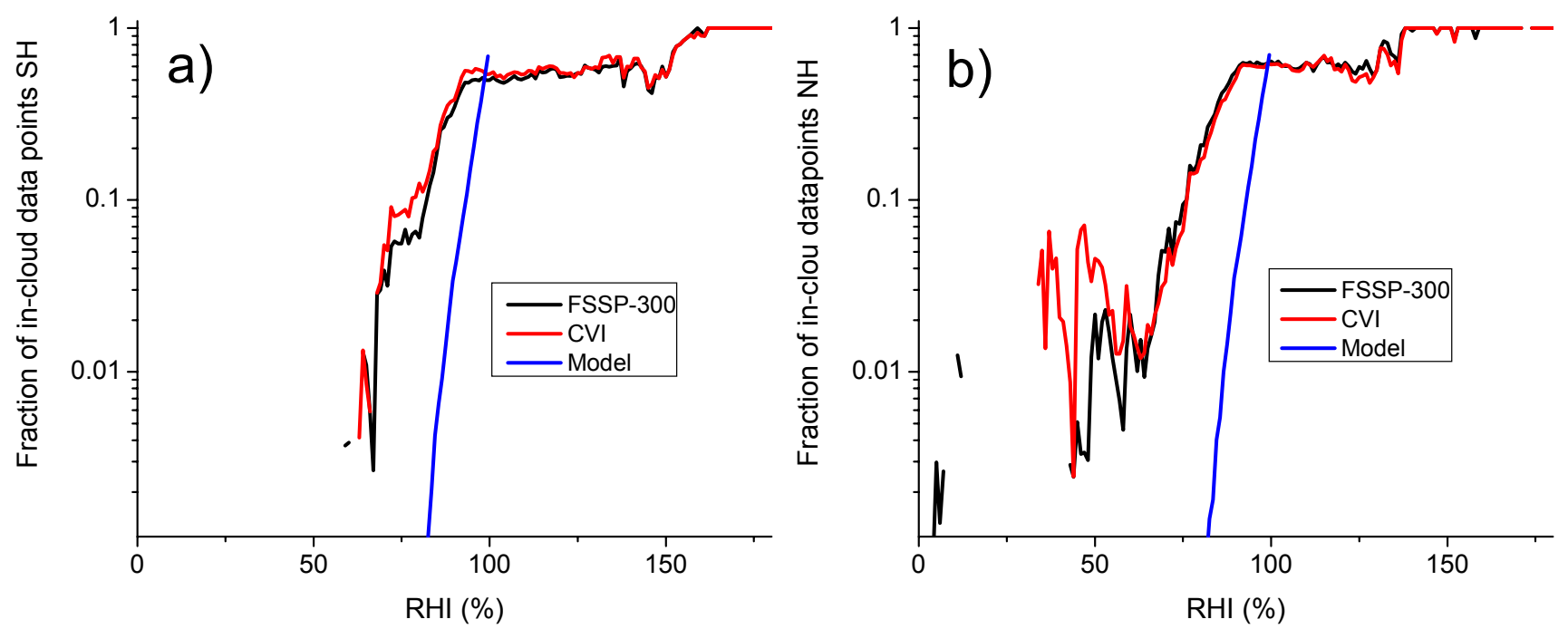

Fig. 2. Observed cloud presence fractions as a function of relative humidity over ice using similar detection thresholds for the two probes (CVI: red curves, FSSP-300: black curves) with respect to particle size $(>4 \mu \mathrm{m})$ and number density $\left(>0.3 \mathrm{~cm}^{-3}\right)$. Data from the SH campaign (a) and from the NH campaign (b). The blue curves are corresponding model results taken below ice saturation and exclusively count ice crystals.

the presence or non-presence of the cloud and the $\mathrm{CPF}=1$ at $\mathrm{X}_{1}$ and humidities above. At $\mathrm{X}_{2}$ (near ice saturation), CPF is less than unity since this point represents a mixture between air parcels that contain cloud particles and where the humidity is perhaps decreasing, and air parcels that have not yet formed a cloud and where the humidity might still be increasing. To the left of $X_{2}$ (below ice saturation), ice crystals begin to evaporate. Once below saturation, all clouds will eventually have disappeared at some humidity $\mathrm{X}_{3}$. Because of the slower evaporation processes in cold clouds the relative humidity at $X_{3}$ is different from $X_{2}$. The details about $\mathrm{X}_{1}, \mathrm{X}_{2}$ and $\mathrm{X}_{3}$ for cirrus clouds are not well known.

\section{Observations}

Our study is limited to observations performed above $6 \mathrm{~km}$ altitude and temperatures below $235 \mathrm{~K}$. Under these conditions, clouds will consist of ice crystals and interstitial aerosol particles (sometimes referred to as supercooled haze droplets), as liquid water droplets would freeze spontaneously. Although the different cloud probes are based on different working principles and have different detection limits, as summarized in Sect. 2.1, it is interesting to compare the consistency between the probes for a subset of the data where there is an overlap in cloud detection. The FSSP-300 is able to detect clouds when particles are smaller than the aerodynamic cut-off of the CVI, but the CVI is able to detect clouds with a much lower particle number density than the FSSP-300 is capable of. If a subset of the FSSP-300 data is selected to emulate a CVI with respect to the size cut-off, and a subset of the CVI data is selected to emulate the FSSP-300 with respect to the number density detection limit, the two instruments should detect clouds with similar efficiency.

\subsection{Transition between clouds and cloud-free air}

Figure 2 shows observed CPFs as a function of RHI from both INCA campaigns ( $\mathrm{SH}$ in Fig. 2a and NH in Fig. 2b) taken with the CVI (red curves) and the FSSP-300 (black curves). To enable a direct comparison, we have used similar detection thresholds for both instruments: particle size $>4 \mu \mathrm{m}$ and particle concentration $>0.3 \mathrm{~cm}^{-3}$. Given these thresholds, we expect the detected particles to be mostly ice crystals, as such high number densities of large aerosol particles are hardly found in cirrus levels (Kärcher and Solomon, 1999). For example, in situ measurements over continental Europe revealed the presence of coarse-mode aerosol in the tropopause region, but only at low concentrations (Schröder et al., 2002, their Fig. 3b). The measured CPFs show more structure than suggested by the schematic in Fig. 1b. We observe a plateau region between $90-130 \%(\mathrm{NH})$ and $90-150 \%$ (SH), a transition region between $70-90 \%$, and a dry region $<70 \%$.

The comparison of CPF data from both campaigns shows an excellent agreement between the CVI and FSSP-300 over several orders of magnitude in CPF. Typically, the difference between the two cloud probes is in the range of percent. The $\mathrm{CPF}$ is plotted on a logarithmic scale to highlight the agreement even down to $\mathrm{RHI}=70-80 \%$. Before the CPFs rapidly decrease around $80 \%$, they stay elevated over a wide range of RHI up to about $130 \%(\mathrm{NH})$ and $150 \%(\mathrm{SH})$. Recall that the CVI counts residual particles from evaporated crystals, whereas the FSSP-300 detects the scattered light from the 

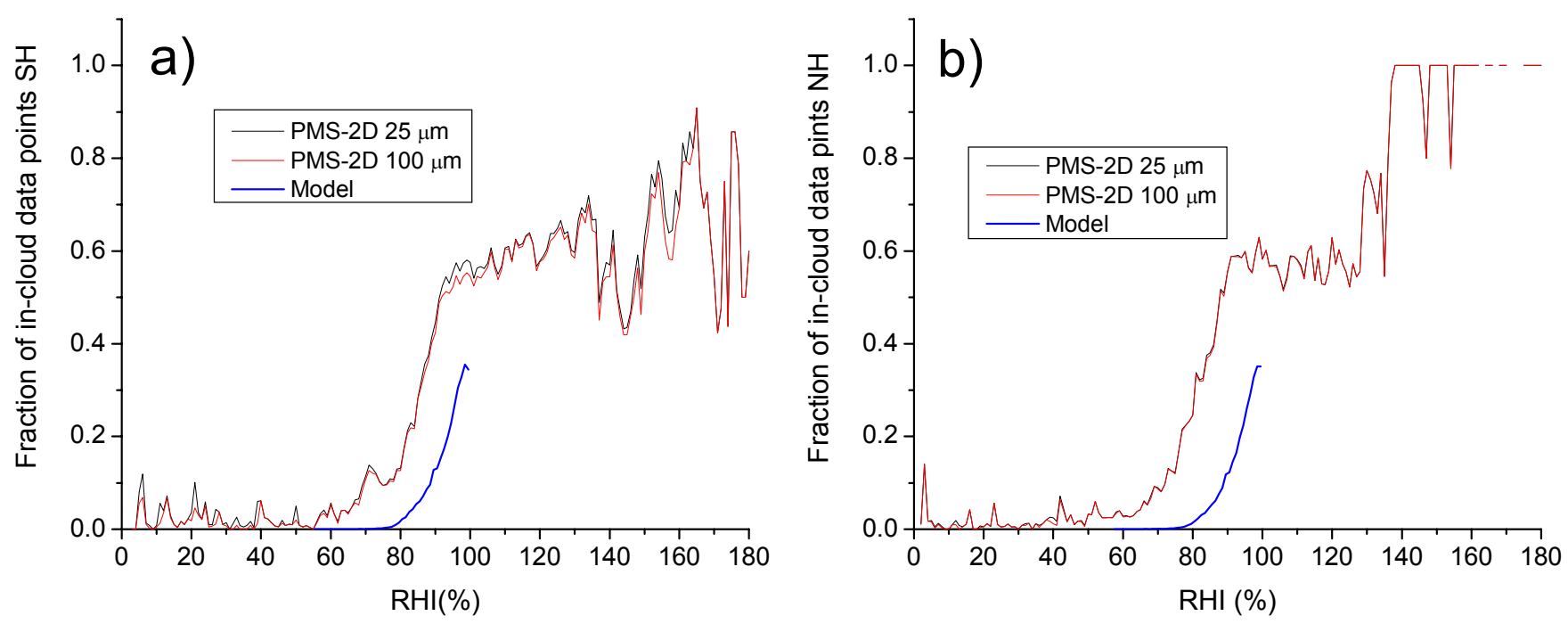

Fig. 3. Cloud presence fractions as a function of relative humidity over ice for different ice crystal size thresholds (black curves: $25 \mu \mathrm{m}$; red curves: $100 \mu \mathrm{m}$ ) as measured by the PMS-2D probe (concentration threshold $10^{-6} \mathrm{~cm}^{-3}$ ). Data from the SH campaign (a) and from the NH campaign (b). Black and red curves in Fig. 5b are almost identical. The blue curves are corresponding model results taken below ice saturation and exclusively count ice crystals $>25 \mu \mathrm{m}$ and $>10^{-6} \mathrm{~cm}^{-3}$.

crystals in the ambient air. Although their working principles are completely different, Fig. 2 proves that the two instruments perform as expected within at least the overlapping range in particle number density and size.

In Fig. 3 the CPFs derived from the 2D-probe are plotted using two cloud presence size thresholds $(25 \mu \mathrm{m}$ and $100 \mu \mathrm{m}$, respectively), as obtained during the SH (Fig. 3a) and $\mathrm{NH}$ campaign (Fig. 3b). In each data set, the two curves are essentially on top of each other, indicating that whenever ice crystals larger than $25 \mu \mathrm{m}$ are present in the cloud, crystals of sizes larger than $100 \mu \mathrm{m}$ are present as well.

The CPF distributions shown in Fig. 3 share many features of those shown in Fig. 2. As for the CVI and FSSP300 probes, we observe a plateau in CPF at RHI between about $90-140 \%$ and a rapid decrease of CPF around $80 \%$. However, for RHI $<70 \%$, the CPFs drop almost to zero in the case of the 2D-probe, strongly indicating that ice crystals with sizes $>25 \mu \mathrm{m}$ do not anymore exist under such subsaturations, while there seem to be values of $\mathrm{CPF}>0$ in the case of the CVI and FSSP-300 probes shown in Fig. 2, especially in the NH data set. We reiterate this issue later in Sect. 3.2.

In what follows, we compare the findings shown in Figs. 2 and 3 with model simulations described in full detail by Haag et al. (2003), whose principal goal is to infer freezing thresholds and nucleation modes from the observed distributions of RHI above ice saturation. Here, it is sufficient to note that the microphysical trajectory model roughly captures the typical environmental conditions (temperatures, cooling rates, mesoscale wave amplitudes and frequencies) that prevailed during the $\mathrm{NH}$ and $\mathrm{SH}$ campaigns and uses a fairly detailed microphysical scheme to predict the formation and disappearence of cirrus clouds. The prescribed aerosol freezing properties provide a consistent explanation of the $\mathrm{SH}$ data in terms of homogeneous freezing, and of the $\mathrm{NH}$ data in terms of homogeneous freezing competing with a small number of efficient ice nuclei (cases HOM and MIX0.001 of Haag et al. (2003), respectively).

We present model-derived CPFs computed from the distributions of RHI inside and outside of cirrus clouds. The calculated CPFs do not include aerosol particles, in contrast to the observations that make no distinction between these two types of particles. They are plotted as blue curves in Figs. 2 and 3, using the same criteria to define cloud as in the observations with the CVI/FSSP-300 and 2D-probe, respectively. The model curves show the same characteristic transition regime as the measurements at roughly comparable RHIs (see below). The reason for the strong decrease of CPF in the model is that the evaporation of water molecules from the ice crystals is kinetically limited. The time needed to (almost) completely evaporate water from ice crystals can be longer than the time in which air parcels experience (significant) subsaturation due to warming, especially at low temperatures and in the presence of temperature oscillations.

Although the model curves are very similar for the SH and $\mathrm{NH}$ cases, they seem to be shifted to the right compared to the observations. We offer four explanations to this apparent discrepancy.

1. The calculated and measured CPF values may differ from each other as, by definition, CPF depends on the ratio between data points taken outside of clouds and 

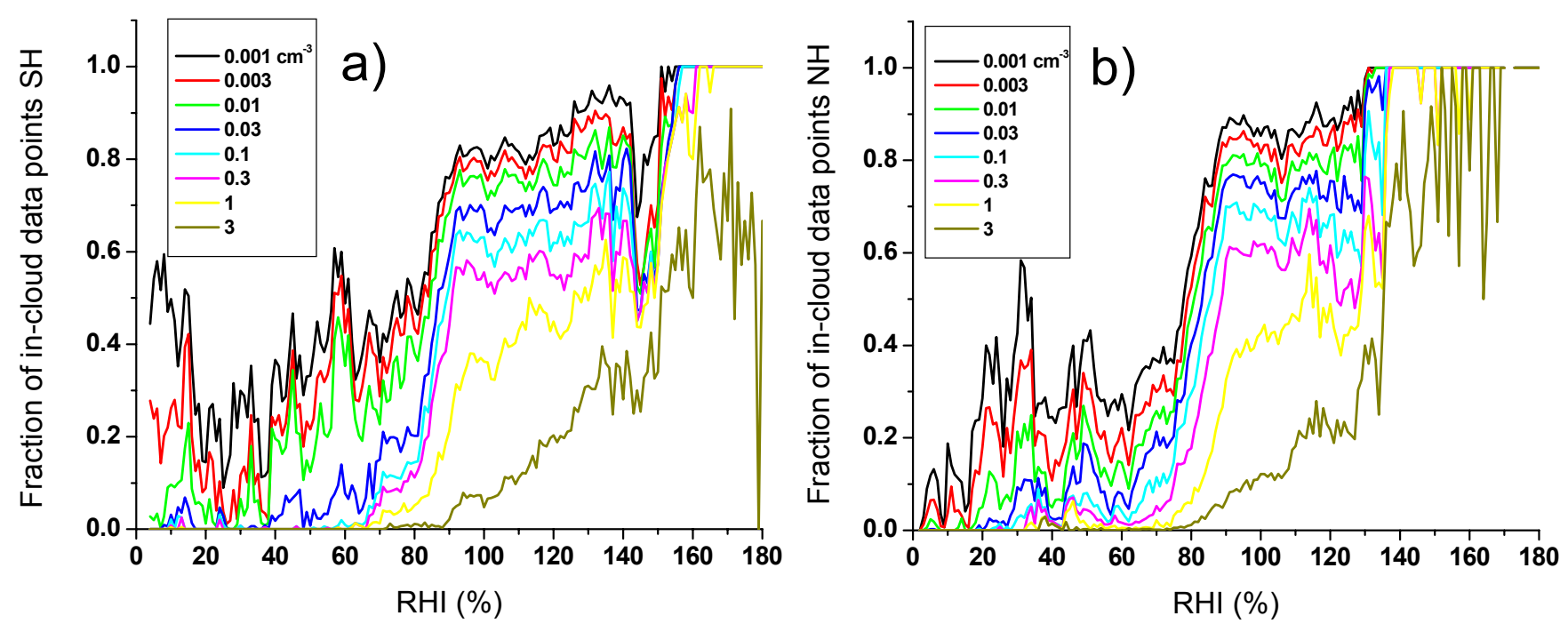

Fig. 4. Cloud presence fractions as a function of relative humidity over ice for different particle number density thresholds (color code given in the legend) as measured by the CVI. Data from the SH campaign (a) and from the NH campaign (b).

those taken inside clouds. Hence, the shifts may in part be artifacts, because this ratio will not be exactly reproduced by the model at any given RHI, and the model CPFs need to be re-scaled as a function of RHI in order to be directly comparable to the observations.

2. The physics of the ice-to-aerosol transition in the model is incomplete. In the current version of the code, we track the ice water mass and compare it with the equilibrium water mass aerosol particles with the same average core (sulfuric acid) mass as contained in these crystals would have at the local thermodynamic conditions. If the ice water mass falls below that value, we remove the corresponding ice particles and reinitialize the aerosol size distribution. A single particle treatment and very small time steps would improve the calculation; more importantly, the chemistry of melting of the ice particles and their chemical composition besides water is not known and does not justify a more detailed description.

3. Small-scale turbulent mixing and entrainment/detrainment processes may affect the evaporation time of small ice crystals but are not represented in the model.

4. The experimental concentration and size thresholds for cloud presence prescribed in the calculations may not be accurate, as we find that the modeled transition region is sensitive to changes in the concentration threshold.

While above the points have no impact on the results presented by Haag et al. (2003), they may at least in part help reconcile data and model results, but important uncertainties remain concerning the evaporation kinetics of small ice crystals.

\subsection{Variation of cloud detection thresholds}

Kärcher and Solomon (1999) have emphasized the continuum aspect of aerosol/cirrus cloud systems in the tropopause region. Several particle types usually coexist, especially in high relative humidity conditions. This notion is strongly supported by the data shown in Figs. 4 and 5 described below. In the size range detectable by the CVI $(>5 \mu \mathrm{m})$, we expect the aerosol/cloud system to be predominantly composed of ice crystals when $\mathrm{CPF}=1$, with increasing contributions from interstitial aerosol particles for $\mathrm{CPF}<1$, especially at RHIs in the vicinity of saturation. (Of course, the total number of aerosol particles regardless of size generally exceeds the number of ice crystals by several orders of magnitude.) Around the point where clouds start to dissolve, the fraction of such large aerosol particles, if present in sufficient concentrations, increases and eventually becomes dominant. Owing to the poorly known kinetics of evaporation of small ice crystals, as mentioned above, the RHI at which the last ice crystals have disappeared is not well defined and may extend to values $<70 \%$. At very dry RHI, well below $70 \%$, we expect the aerosol/cloud system to be exclusively composed of aerosol particles, some of which may exist in crystalline or mixed states. Although the term "cloud" does not actually apply in such very dry situations, we use the term CPF over the entire range of RHI to support our claim that there is no universal or well defined limit in RHI below which we always find "pure aerosol". The only objective measure available to us is the detection limit of the sensors we use. 

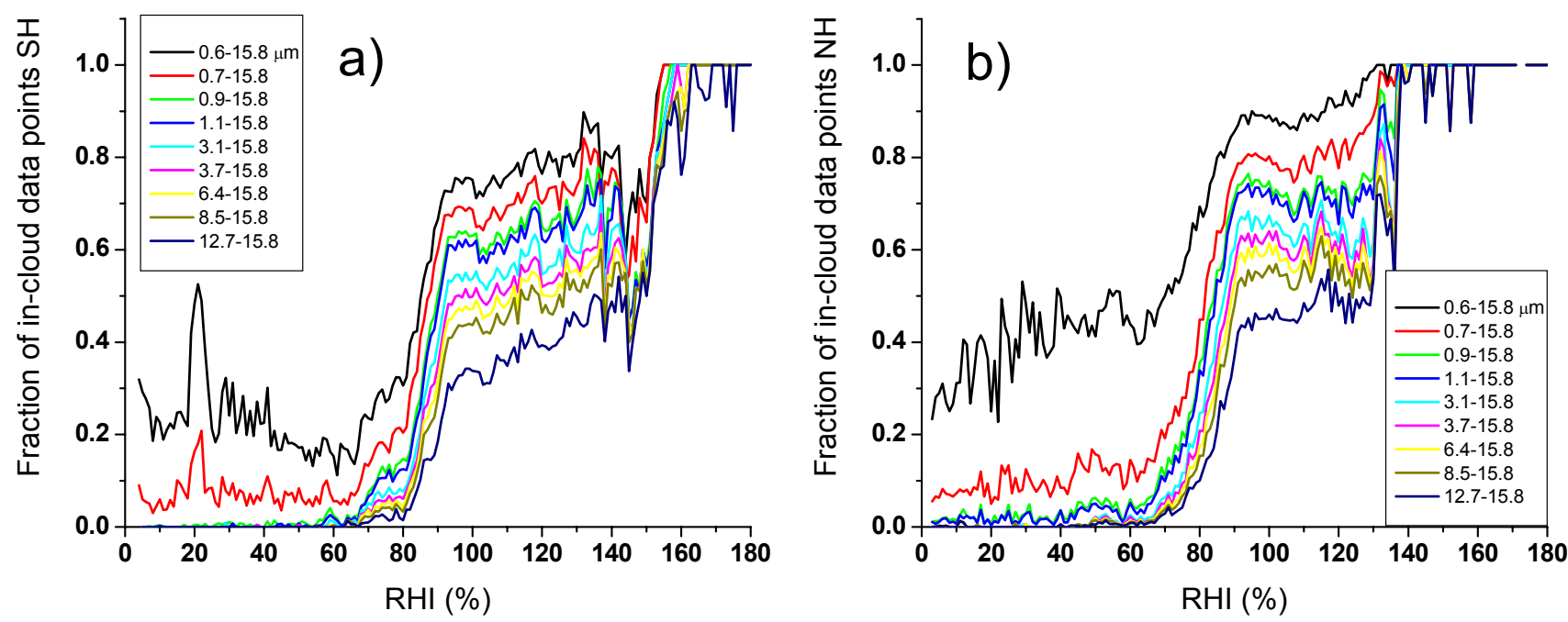

Fig. 5. Cloud presence fractions as a function of relative humidity over ice for different particle size thresholds (color code given in the legend) as measured by the FSSP-300. Data from the SH campaign (a) and from the NH campaign (b).

We will use data from the CVI to study in more detail the presence or non-presence of clouds based on different number density thresholds, and the FSSP-300 using different size thresholds. In Fig. $4 \mathrm{CPF}$ is plotted versus RHI using different number density thresholds from the CVI for the $\mathrm{SH}$ (Fig. 4a) and NH (Fig. 4b) campaign, respectively. For all but the highest threshold $\left(3 \mathrm{~cm}^{-3}\right)$ there is a sharp drop in CPF between $70-90 \%$ RHI, marking the transition region between cloudy and cloud-free air, as motivated in Sect. 3.1.

In the plateau region between $90 \%$ and $130 \%(\mathrm{NH})$ or $150 \%(\mathrm{SH})$, the CPF increases with increasing sensitivity (decreasing concentration threshold) to measure cloud particles which are essentially ice crystals given the lower size cut-off of the CVI. Note that the CPFs from both data sets become rather small for the highest concentration threshold $\left(3 \mathrm{~cm}^{-3}\right)$ and do not even reach unity at the highest RHI. This is explained by the fact that we now begin to miss most of the ice crystals, because the threshold of $3 \mathrm{~cm}^{-3}$ is of the same order as the average total number of ice crystals observed in cirrus during both INCA campaigns (Kärcher and Ström, 2003).

In the dry region, $\mathrm{RHI}<70 \%, \mathrm{CPF}$ is almost zero for concentration thresholds $>0.3 \mathrm{~cm}^{-3}$, indicating that cloud particles (i.e., either aerosol or ice particles) with sizes $>5 \mu \mathrm{m}$ are not present with such high number densities. As discussed in Sect.3.1, it is not entirely clear from the data how far away from the transition region towards low RHI ice crystals may still exist (and form a "cloud"). To say at which RHI an ice crystal becomes an aerosol particle is difficult; at any rate, the fraction of aerosol particles will certainly increase with decreasing RHI. Consequently, as we relax the threshold concentration to values $<0.3 \mathrm{~cm}^{-3}$, the CVI detects large aerosol particles down to very dry conditions in up to $\sim 40 \%$ of all measurements at concentration thresholds $<0.01 \mathrm{~cm}^{-3}$.
In Figs. 5a and b CPF is plotted using different size thresholds from the FSSP-300 for the SH and NH data set, respectively. In selecting the size thresholds, we have used the available size bin classifications and do not distinguish between different particles types, i.e., dry particles, haze droplets, or ice crystals.

The principal features of CPF are similar to Fig. 4. In particular, the transition region is located around $80 \%$ RHI, as before. However, given the detection limit of $0.2 \mathrm{~cm}^{-3}$, we are mostly detecting ice particles with the FSSP-300 when using the size thresholds $>3.7-6.4 \mu \mathrm{m}$. Using lower size cutoffs results in the enhanced detection of (interstitial) aerosol particles and ice crystals, and makes the interpretation of the curves as true cloud presence fractions more difficult.

A notable difference between the NH and SH data sets occurs in the dry region between the size thresholds $0.6 \mu \mathrm{m}$ and $0.7 \mu \mathrm{m}$. During the NH campaign particles in the size range $0.6-0.7 \mu \mathrm{m}$ was present twice as often as during the $\mathrm{SH}$ campaign. This perhaps indicates a difference in the presence of ice nuclei between the two measurement locales or a difference in evaporation kinetics.

\subsection{Onset of freezing in cirrus clouds}

In Fig. 6, cloud data points are defined as having an extinction coefficient of $0.05 \mathrm{~km}^{-1}$ or larger as measured by the Polar Nephelometer. In addition, this criterion had to be fulfilled during at least four consecutive seconds in order to be considered an in-cloud data point. A microphysical equivalent of this optical threshold corresponds to ice crystals of $\sim 5 \mu \mathrm{m}$ diameter at a number density roughly between 0.05 and $0.1 \mathrm{~cm}^{-3}$. The criteria used to define Polar Nephelometer in-cloud data points presented in Fig. 6 are the same as used by Ovarlez et al. (2002). Whereas all previous CPFs 


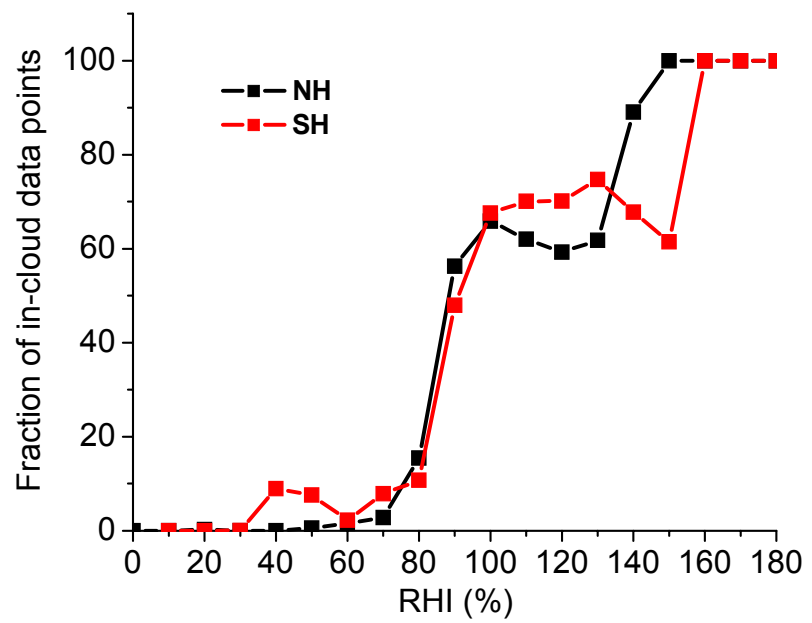

Fig. 6. Cloud presence fractions as a function of relative humidity over ice measured by the Polar Nephelometer. If the probe, at a given RHI, observes an extinction coefficient exceeding $0.05 \mathrm{~km}^{-1}$ during four consecutive seconds, the data point is considered a cloudy data point. This criterion roughly corresponds to particle size and concentration thresholds of $5 \mu \mathrm{m}$ and $0.05-0.1 \mathrm{~cm}^{-3}$, respectively, indicating that mostly ice crystals are detected. Only data during straight and level flight segments are included.

were calculated for each percent of RHI, the Polar Nephelometer data are averages over $10 \%$ increments.

Values of CPF near zero in the dry region below $70 \%$ support the notion that the Polar Nephelometer has exclusively probed ice crystals. (The origin of the hump at RHI between $40-50 \%$ in the SH data set is not known.) In contrast to what is expected from the schematic shown in Fig. 1b, however, the CPF curves in Fig. 6 do not increase monotonically to unity when RHI rises, but exhibit local minima centered at $120 \%$ (NH data) and 150\% (SH data). This feature can also be traced in the data of the other probes presented in Figs. 25 , but the extent of the minimum depends on the threshold used to characterize in-cloud and out-of-cloud data points.

As the Polar Nephelometer detects ice crystals in cirrus when they return an extinction signal $>0.05 \mathrm{~km}^{-1}$, a sufficient number of crystals must have nucleated and grown to certain sizes in order to produce such large extinction values. The very first, freshly nucleated crystals are certainly not detectable by the Polar Nephelometer probe. When all ice crystals have formed at RHI $>130 \%(\mathrm{NH})$ and $>150 \%$ (SH), they grow and thereby reduce RHI and become detectable by the Polar Nephelometer when crossing again the RHI-regions containing the local minima. The same reasoning can be applied to the other probes.

This provides an explanation for the distinct minima observed in the Polar Nephelometer data presented in Fig. 6. Thus, the interpretation of the local minimum in observed $\mathrm{CPF}$ above ice saturation is that it approximates the range in RHI where the onset of cloud formation occurs. The compar- ison of the $\mathrm{NH}$ and $\mathrm{SH}$ cases strongly suggests that the mode of nucleation was different during the respective campaigns.

The study of Haag et al. (2003) investigates this difference in ice nucleation in cirrus in more detail by analyzing the distributions of RHI taken outside of and inside cloud with the help of microphysical model simulations.

\subsection{Cloud water content}

Figure 7a shows the observed cloud water contents derived from the sum of the CWC's measured by the 2D-probe using an empirical formulation (Gayet et al., 2002) to convert from size distribution to CWC, and the CWC derived from the FSSP-300 size distribution assuming spherical particles and using a particle mass density of $0.9 \mathrm{~g} \mathrm{~cm}^{-3}$. The observed distributions are normalized, which makes the areas under the curves equal to unity.

Both NH (black curve) and SH (red curve) distributions of CWC are rather similar and are skewed with a tail towards lower CWCs. The calculated geometric mean CWCs are 7.3 and $10.4 \mathrm{mg} \mathrm{m}^{-3}$ for the $\mathrm{SH}$ and $\mathrm{NH}$ data, respectively. The data points below $0.01 \mathrm{mg} \mathrm{m}^{-3}$ are almost entirely data from the FSSP-300 when the 2D-probe did not register any crystals, hence aerosol particles and perhaps the smallest ice crystals contribute to such low CWCs. The water vapor available to convert to CWC depends strongly on temperature, we therefore derive a similar CWC distribution taken in a narrow temperature range between 225 and $227 \mathrm{~K}$, as presented in Fig. 7b. The distributions in Figs. 7a and b essentially exhibit the same features, but the mode or maximum of the distributions for the more narrow temperature range is more defined.

The rather small differences in the distributions of CWC between the SH and $\mathrm{NH}$ campaigns along with the fact that the distributions of temperatures, updraft speeds, and total ice crystal concentrations were also quite similar suggests that existing differences in the freezing properties of aerosol particles between $\mathrm{SH}$ and $\mathrm{NH}$ data have hardly affected the CWC. Rather the CWCs appear to be mainly determined by dynamical factors such as the advection of water vapor controlling the availability of condensable water.

\section{Conclusions}

In this study we employed different cloud probes to determine the presence or non-presence of cirrus clouds as function of relative humidity over ice during the INCA campaigns. The observed cloud presence fractions showed a characteristic behavior when plotted as a function of relative humidity over ice: a flat, plateau-like region between RHI $=90 \%$ and values close to the cloud formation thresholds above which $\mathrm{CPF}=1$; a transition region between $70-90 \%$ where the bulk of the ice crystals evaporate and the cloud 

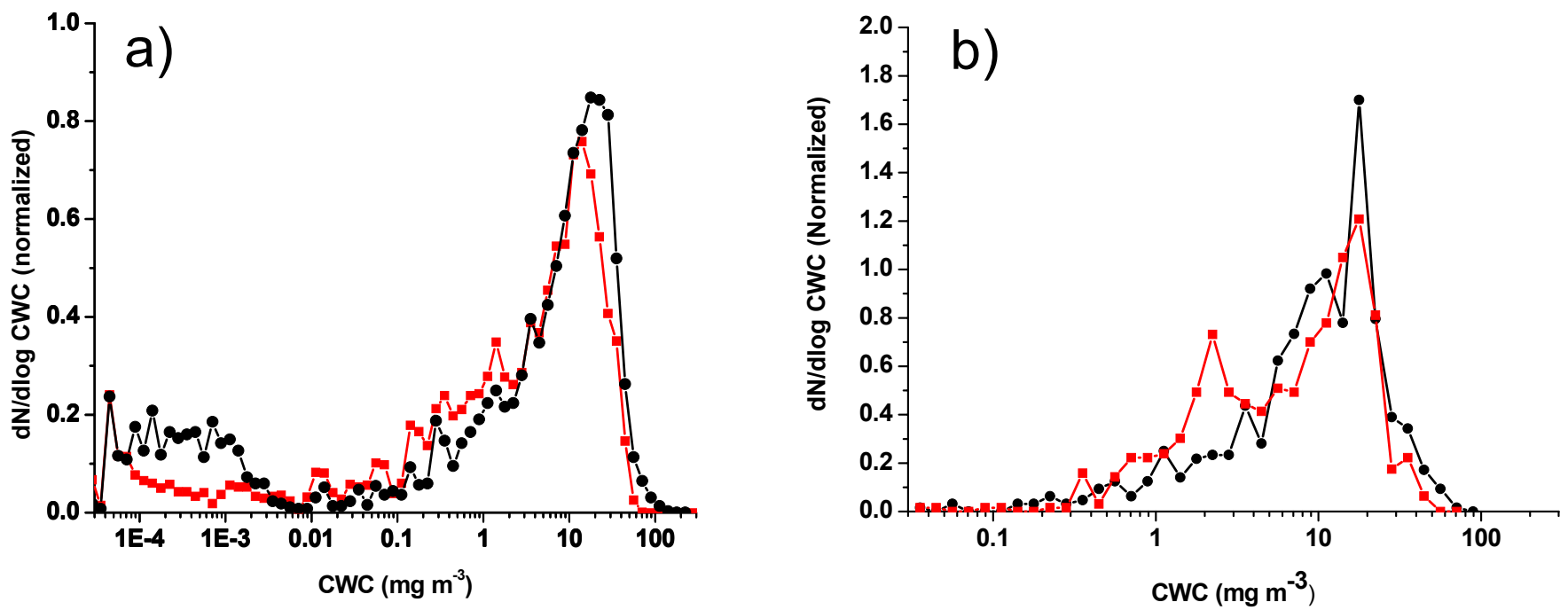

Fig. 7. Observed distributions of cloud water content (SH: red curves, NH: black curves). In (a) all temperatures below $235 \mathrm{~K}$ and relative humidities between 95 and 105\% RHI are used. In (b) the temperature only ranges between 225 and $227 \mathrm{~K}$. The observed CWC is based on the sum of the FSSP-300 and 2D-probe data. Note the different scaling of the CWC axes.

dissolves; and a dry particle region $<70 \%$ where eventually all ice particles disappear.

The CPF derived from the Polar Nephelometer data exhibits a distinct local minimum between $140-155 \%$ RHI in the SH data set when plotted as a function of RHI. A less pronounced local minimum in CPF is also suggested in the $\mathrm{NH}$ data set between approximately $115-130 \%$ RHI. Our interpretation of these features is that they correspond to the preferred ice nucleation thresholds during the respective campaigns. The SH threshold is consistent with homogeneous freezing, whereas the $\mathrm{NH}$ threshold indicates heterogeneous ice formation. A comprehensive analysis of this finding is presented in Haag et al. (2003).

Our data strongly support the notion that (interstitial) aerosols and cirrus clouds form a continuum system. As we have shown, how well we can experimentally distinguish between ice crystals, haze droplets, and other aerosol particles as a function of RHI will largely depend on the sensors used to probe the aerosol/cirrus system. The CPFs describe "pure aerosol" below a poorly defined RHI $<70 \%$, but never really describe "pure cloud" inasmuch as interstitial aerosol particles are not completely scavenged by the ice crystals during the cloud lifetime. The evaporation kinetics of small ice crystals and the haze particle properties above ice saturation are poorly constrained by observations and must remain under scrutiny to fully understand how cirrus clouds form and disappear in the atmosphere. In this regard, there is a need for a measurement device combining the size detection sensitivity of the FSSP-300 and the concentration detection sensitivity of the CVI, and for size-resolved in situ chemical characterization of aerosol and ice particles.
Acknowledgements. This work was funded by the European Commission through the projects INCA and PARTS. It also contributes to the project "Particles and Cirrus Clouds" (PAZI) supported by the Helmholtz-Gemeinschaft Deutscher Forschungszentren (HGF). The Swedish Research Council is sponsoring this work by supporting ITM in airborne aerosol and cirrus activities. We thank the entire INCA team for help in collecting the data.

\section{References}

Auriol, F., Gayet, J.-F., Febvre, G., Jourdan, O., Labonnotte, L., and Brogniez, G.: In situ observations of cirrus cloud scattering phase function with $22^{\circ}$ and $46^{\circ}$ halos: Cloud field study on 19 February 1998, J. Atmos. Sci., 58, 3376-3390, 2000.

Baumgardner, D., Dye, J. E., Gandrud, B. W., and Knollenberg, R. G.: Interpretation of measurements made by the forward scattering spectrometer probe (FSSP-300) during the Airborne Arctic Stratospheric Expedition, J. Geophys. Res., 97, 8035-8046, 1992.

Borrmann, S., Luo, B. P., and Mishchenko, M.: Application of the T-matrix method to the measurement of aspherical (ellipsoidal) particles with forward scattering optical particle counters, J. Aerosol Sci., 31, 789-799, 2000.

Boucher, O.: Air traffic may increase cirrus cloudiness, Nature, 397, 30-31, 1999.

Chen, J.-P. and Crutzen, P. J.: Solute effects on the evaporation of ice particles, J. Geophys. Res., 99, 18 847-18 859, 1994.

DeMott, P. J.: Laboratoty studies of cirrus cloud processes, in: Cirrus, edited by Lynch, D. K., et al., Oxford Univ. Press, New York, pp.102-135, 2002.

European Commission (EC): European research in the stratosphere 1996-2000, Second assessment on stratospheric research, edited by Amanatidis, G. T. and Harris, N. R. P., 257-307, 2002. 
Gayet, J.-F., Brown, P. R., and Albers, F.: A comparison of in-cloud measurements obtained with six PMS 2D-C probes, J. Atmos. Ocean. Technol., 10, 180-194, 1993.

Gayet, J.-F., Crépel, O., Fournol, J. F., and Oshchepkov, S.: A new airborne Polar Nephelometer for the measurements of optical and microphysical cloud properties. Part I: Theoretical design, Ann. Geophys., 15, 451-459, 1997.

Gayet, J.-F., Auriol, F., Minikin, A., Ström, J., Seifert, M., Krejci, R., Petzold, A., Febvre, G., and Schumann,U.: Quantitative measurement of the microphysical and optical properties of cirrus clouds with four different in situ probes: Evidence of small ice crystals, Geophys. Res. Lett., 29, 2230-2234, 2002.

Haag, W., Kärcher, B., Ström, J., Minikin, A., Ovarlez, J., Lohmann, U., and Stohl, A.: Freezing thresholds and cirrus cloud formation mechanisms inferred from in situ measurements of relative humidity, Atmos. Chem. Phys., 3, 1791-1806, 2003.

Intergovernmental Panel on Climate Change (IPCC), 1999: Aviation and the Global Atmosphere, edited by Penner, J. E., Lister, D. H., Griggs, D. J., Dokken, D. J., and McFarland, M., Cambridge Univ. Press, Cambridge, UK, pp. 373, 1999.

Kristensson, A., Gayet, J.-F., Ström, J., and Auriol, F.: In situ observations of a reduction in effective crystal diameter in cirrus clouds near flight corridors, Geophys. Res. Lett., 27, 681-684, 2000.

Kärcher, B. and Solomon, S.: On the composition and optical extinction of particles in the tropopause region, J. Geophys. Res., 104, 27 441-27 459, 1999.

Kärcher, B. and Lohmann, U.: A parameterization of cirrus cloud formation: Heterogeneous freezing, J. Geophys. Res., 108, 4402, doi:10.1029/JD2002003220, 2003.

Kärcher, B. and Ström, J.: The roles of dynamical variability and aerosols in cirrus cloud formation, Atmos. Chem. Phys., 3, 823838, 2003.

Mannstein, M., Meyer, R., and Wendling, P.: Operational detection of contrails from NOAA-AVHRR-data, Int. J. Remote Sensing, 20, 1641-1660, 1999.

Meyer, R., Mannstein, H., Meerkötter, R., Schumann, U., and Wendling, P.: Regional radiative forcing by line-shaped contrails derived from satellite data, J. Geophys. Res., 107, doi:10.1029/2001JD000426, 2002.
Ogren, J. A., Heintzenberg, J., and Charlson, R. J.: In-situ sampling of clouds with a droplet to aerosol converter, Geophys. Res. Lett., 12, 121-124, 1985.

Ovarlez, J. and van Velthoven, P.: Comparison of water vapor measurements with data retrieved from ECMWF analyses during POLINAT experiment, J. Appl. Meteor., 105, 1329-1335, 1997.

Ovarlez, J., van Velthoven, P., Sachse, G., Vay, S., Schlager, H., and Ovarlez, H.: Comparison of water vapor measurements from POLINAT 2 with ECMWF analyses in high-humidity conditions, J. Geophys. Res., 105, 3737-3744, 2000.

Ovarlez, J., Gayet, J.-F., Gierens, K., Ström, J., Ovarlez, H., Auriol, F., Busen, R., and Schumann, U.: Water vapour measurements inside cirrus clouds in Northern and Southern hemispheres during INCA, Geophys. Res. Lett., 29, 1813-1817, 2002.

Schröder, F., Kärcher, B., Fiebig, M., and Petzold, A.:Aerosol states in the free troposphere at northern midlatitudes, J. Geophys. Res., 107, 8126, doi:10.1029/2000JD000194, 2002.

Seifert, M., Ström, J., Krejci, R., Minikin, A., Petzold, A., Gayet, J.-F., Schlager, H., Ziereis, H., Schumann, U. and Ovarlez, J.: Aerosol-cirrus interactions: A number based phenomenon at all?, Atmos. Chem. Phys., 3, in press, 2003a.

Seifert, M., Ström, J., Krejci, R., Minikin, A., Petzold, A., Gayet, J.F., Schumann, U., and Ovarlez, J.: In situ observations of aerosol particles remaining from evaporated cirrus crystals: Comparing clean and polluted air masses, Atmos. Chem. Phys., 3, 10371049, 2003b.

Sonntag, D.: Advancements in the field of hygrometry, Meteorol. Z., 3, 51-66, 1994.

Ström, J., Heintzenberg, J., Noone, K. J., Noone, K. B., Ogren, J. A, Albers, F., and Quante, M.: Small crystals in cirrus clouds: their residue size distribution, cloud water content, and related cloud properties, J. Atmos. Res., 32, 125-141, 1994.

Ström, J. and Ohlsson, S.: In situ measurements of enhanced crystal number densities in cirrus clouds caused by aircraft exhaust, J. Geophys. Res., 103, 11 355-11361, 1998. 